\title{
Renal complications in thalassemia
}

\author{
Joseph Sleiman, Ali Tarhini, Ali T. Taher \\ Department of Internal Medicine, American University of Beirut Medical Center, Beirut 11-0236, \\ Lebanon
}

\begin{abstract}
Thalassemia is a disease with an extensive morbidity profile affecting almost every organ system. Renal involvement, once considered rare, is an underestimated and poorly studied complication that has been on the rise ever since medical advances granted patients longer life spans. Several studies and reports have emerged recently to shed light on the seriousness of this complication, although data is still lacking in terms of pathophysiology, diagnosis, prevention and treatment. In this review, we evaluate and compare renal involvement in the transfusion-dependent and independent variants of $\beta$-Thalassemia, highlighting the pathophysiology of kidney damage that involves iron overload, chronic anemia, and iron chelation therapy. An in-depth and focused review of the types of injuries incurred is also presented along with the diagnostic biomarkers accompanying each type of injury. Most research so far has focused on the transfusion-dependent thalassemia population being the group with most renal involvement, however recent reports have shown evidence of comparable, if not worse, involvement of the non-transfusion dependent population, sometimes leading to end-stage renal disease. As such, we try to shed light on distinct renal involvements in NTDT whenever available.
\end{abstract}

\section{Introduction}

The thalassemias are common monogenic disorders present worldwide. This inherited disorder of hemoglobin synthesis leads to an imbalance in $\alpha / \beta$-globin production, which manifests as a chronic hemolytic anemia, ineffective erythropoiesis, and iron

Correspondence: Ali T. Taher, Tel.: +961-1-350-000; Fax: +961-1-370-814. E-mail: ataher@aub.edu.lb

Key words: Thalassemia; renal complications; iron chelation therapy; renal biomarkers.

Author contributions: Joseph Sleiman and Ali Tarhini performed research and wrote the paper. Ali T. Taher critically reviewed the manuscript and supervised the whole work. All authors read and approved the final draft.

Conflicts of interest: Joseph Sleiman and Ali Tarhini have no conflicts of interest to disclose. Ali T. Taher receives research funding and honoraria from Novartis Pharmaceuticals, and research funding from Celgene and Roche.

This work is licensed under a Creative Commons Attribution 4.0 License (by-nc 4.0).

CCopyright J. Sleiman et al., 2018

Licensee PAGEPress, Italy

Thalassemia Reports 2018; 8:7481

doi:10.4081/thal.2018.7481 overload (IOL). Clinical presentation ranges from lifelong need of blood transfusions, to careful observation with minimal transfusions, to asymptomatic carriage. These are respectively coined transfusion-dependent thalassemia (TDT), non-transfusion-dependent thalassemia (NTDT), and thalassemia minor. However, with both TDT and NTDT, there exists a multimorbidity profile that stems either from the disease progression itself or from its long term managements (blood transfusions and iron chelators). ${ }^{1}$

The thalassemias have long been diseases of the pediatric population; nowadays, these inherited disorders present an equally significant burden among the adult population. The advances in both the knowledge and the care of this disease, as well as the increase in availability of treatments for those in need, have been factors for the aging of the thalassemic population. Such a change in demographics, however, allowed the recognition of complications associated with the longevity of patients living with thalassemia. ${ }^{2,3}$ Long-term damage to the renal system has become evident with more studies targeting renal biomarkers and prevalence of different types of renal injuries in thalassemia. We discuss herein the renal complications found in thalassemia along with the role of iron overload, chronic anemia and iron chelation therapy (ICT) in their pathophysiology. The bulk of the literature tackles transfusion-dependent thalassemia patients, but information will also be presented on patients with non-transfusiondependent thalassemia.

\section{Methods}

Publications from potentially relevant journals were found on Medline and PubMed through advanced search option with "(thalassemia) AND renal". We excluded articles that cover other hemoglobinopathies unless they invoke a relevance to thalassemia. References of these articles were browsed and more articles were used if they improve understanding of the different aspects of the paper.

\section{Mechanisms of renal complications}

Chronic anemia, iron overload, and the use of specific iron chelators have all been linked to renal manifestations in patients with $\beta$-Thalassemia (Figure 1). ${ }^{4}$ Although some studies on renal function in $\beta$-Thalassemia exist before ICT was adopted, the independent contribution to renal abnormalities by either chronic anemia or iron overload is harder to pinpoint, given their coexistence in the pathophysiology of the disease. . $^{1,2,5,6}$ Other factors that can exaggerate this decrease in the thalassemic population may be glomerular diseases associated with hepatitis B or C and HIV infections (transfusion related), as well as iron-induced hepatic and cardiac dysfunction. ${ }^{6}$

\section{Iron overload}

Different mechanisms of toxicity from local iron in the kidneys are suggested. The nephrons may be exposed to heme and hemecontaining proteins when the body is oversaturated with iron con- 
taining elements from persistent hemolysis. Blood transfusions can further dump both non-transferrin bound iron (NTBI) and heme elements into the nephrons. Autopsy series on patients with TDT showed iron deposition in terminal portions of the proximal tubules and in the distal tubules. ${ }^{7}$ Such hemosiderin deposits may pave the way for tubular necrosis, cortical atrophy, and interstitial fibrosis, which could be a factor in both acute and chronic kidney injuries in thalassemia. Iron itself plays a role in worsening the progression of chronic kidney disease (CKD), as studies on CKD individuals without states of iron overload showed a deceleration in CKD progression with the use of iron chelators. ${ }^{8}$

Iron is speculated to dissociate from transferrin in the acidic milieu of the proximal tubules, producing reactive oxygen species (ROS) that can damage the brush border of the renal tubular membrane. Even transferrin bound iron that enters the proximal tubular cells can still cause cellular damage by creating ROS once free iron is released upon lysosomal digestion. ${ }^{9,10}$ Mitochondrial stress is linked to the resulting cellular injury, as it appears that an increased release of cytochrome $\mathrm{C}$, lactate dehydrogenase and reduction in adenosine triphosphate (ATP) are noticed in damaged proximal tubular cells. ${ }^{11}$ Oxidants also increase the susceptibility of glomerular basement membranes to damage by decreasing synthesis of proteoglycans, necessary for its integrity. ${ }^{12}$

In addition, tubulointerstitial damage may result when growth factors and cytokines are released from injured tubular cells, creating a bed for tubulointerstitial fibrosis and glomerular sclerosis. ${ }^{13}$

\section{Chronic anemia}

Renal tubular cells can equally be exposed to oxidative stress and lipid peroxidation from states of chronic hypoxia and anemia, even without iron overload. ${ }^{14}$ The severity of anemia correlated well with markers of tubular abnormalities; the latter were reduced in the thalassemia major group with hypertransfusion regimens. ${ }^{15}$ Mixed results however do exist. ${ }^{16}$ Renal manifestations in NTDT patients who are ICT naïve could thus be the result of the interplay between chronic anemia and iron overload.

It is suggested that the decrease in systemic vascular resistance in the anemic state can instigate the increased renal plasma flow and subsequently the glomerular filtration rate (GFR), resulting in what is known as "glomerular hyperfiltration". ${ }^{17}$ Such a phenomenon, reflected clinically as an increase of creatinine clearance $(\mathrm{CrCl})$ or estimated GFR (eGFR), is usually associated with diabetes, hypertension or their precursor states. It brings about a state of glomerular hypertension, where glomerular capillary walls stretch and endothelial/epithelial injury ensues. Macromolecular leaks occur to relieve the intraglomerular pressure, but end up causing glomerulo-tubular dysfunctions like increased albuminuria and may lead to end-stage renal disease (ESRD). ${ }^{18,19}$

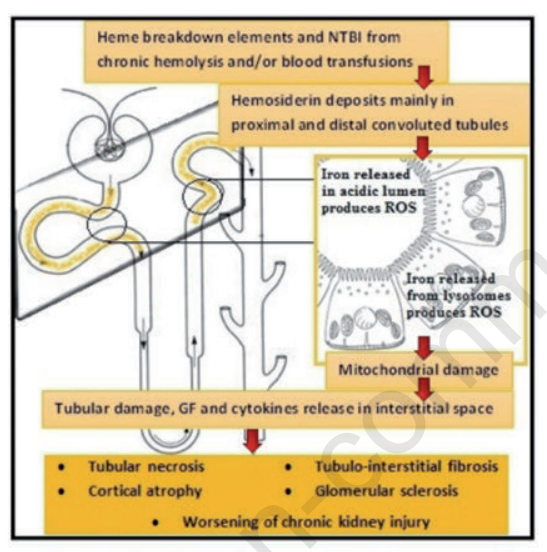

A. Iron Overload

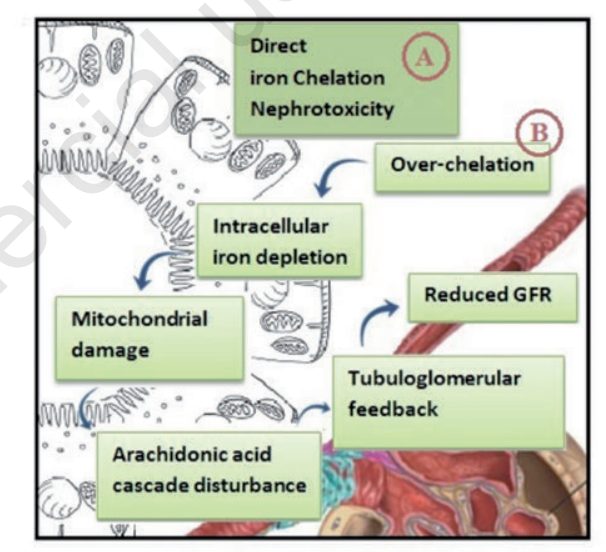

B. Iron Chelation Therapy

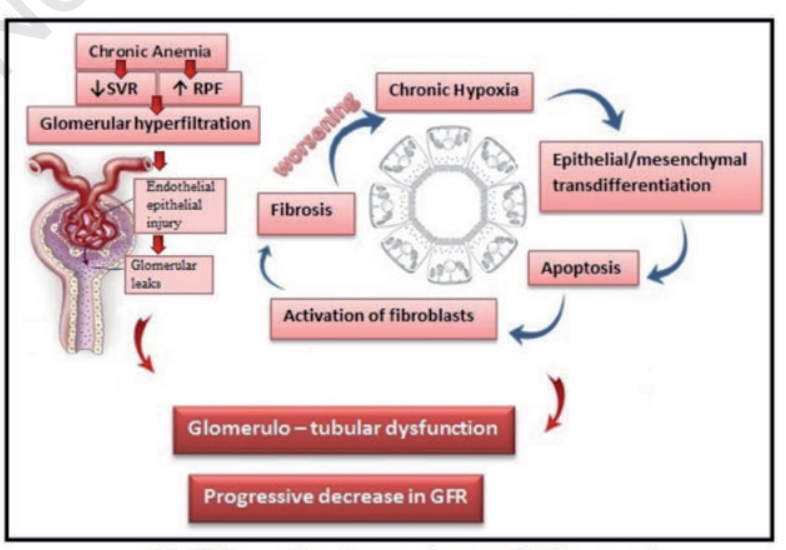

C. Chronic Anemia and Hypoxia

Figure 1. Pathophysiology behind the renal complications in thalassemia. (a) the suggested pathophysiology of renal disease due to iron overload; (b) two possible mechanisms for renal complications due to iron chelation therapy; (c) the impact of chronic anemia and hypoxia on kidney structure and function. NTBI, non transferrin bound iron; ROS, reactive oxygen species; GF, growth factor; GFR, glomerular filtration rate; SVR, systemic vascular resistance; RPF, renal plasma flow. 
On the other hand, chronic hypoxia can throw tubular cells, whose metabolic demands are physiologically high, into a vicious cycle of epithelial-mesenchymal transdifferentiation, apoptosis, activation of fibroblasts and subsequent fibrosis. Fibrosis of the interstitial space then worsens oxygen diffusion, further propagating the tubular damage that was initiated by hypoxia. The aftermath can vary between tubulointerstitial injury, glomerulosclerosis and kidney fibrosis, especially in the setting of iron overload. ${ }^{4,13,20}$ GFR would eventually decrease after prolonged cellular injury, possibly ending in ESRD. ${ }^{19-22}$

\section{Iron chelation therapy}

Management of iron overload in both forms of $\beta$-Thalassemia comes at an expense. Three agents are available for iron chelation - oral deferiprone (DFP), deferasirox (DFX), and the parenteral deferoxamine (DFO) - each with a spectrum of side effects. Kidney injury is a prominent side effect with DFX and DFO, especially when tight dosage monitoring is absent. Increased urinary excretion of beta-2 microglobulin and growth hormone after continuous DFO infusion suggests a role for over-chelation. ${ }^{23}$ Direct nephrotoxic effect via tubular necrosis is the most likely mechanism of kidney injury according to biopsy findings and due to the reversible nature of the injury. ${ }^{24}$ Additionally, over-chelation and depletion of serum iron has been hypothesized to play a role in renal damage. This has been supported by a trend of increasing serum creatinine in groups who showed significant decreases in liver iron concentration (LIC) and serum ferritin (SF) or in those with initially lower baseline iron indices. ${ }^{4,25,26}$ Relative intracellular iron depletion is also theorized to trigger an imbalance between vasodilating and vasoconstrictive prostaglandins. This is due to tubular cell mitochondrial damage, reduction in ATP production and interference with arachidonic acid cascade, which can ultimately alter prostaglandin production and trigger tubuloglomerular feedback in favor of reduced GFR. ${ }^{4}$

\section{Types of renal Injury}

Kidney dysfunctions in thalassemia, namely TDT, tend to increase with age and correlate with risk factors (e.g. blood transfusion duration) or renal function biomarkers (e.g. hypercalciuria) ${ }^{27}$ Many aspects of kidney can be affected, and these often coexist in the same patients. ${ }^{28}$ More is known about TDT renal complications than NTDT at this point, as renal manifestations in NTDT patients are poorly described and data on the subject are scarce with lack of long-term follow-ups. So far, most studies on renal manifestations in this subgroup have revolved around the potential nephrotoxic effects of iron chelators. ${ }^{4,29}$ It is very clear, however, that NTDT deserves comparable attention when it comes to renal outcomes. ${ }^{30}$

\section{Tubular injury}

There is evidence for tubular damage in the TDT population. Almost all patients are found to have low-molecular weight proteinuria at some point in time. Urinary markers of proximal tubular damage have been increased in several studies; these include $\mathrm{N}$ acetyl- $\beta$ - $D$-glucosaminidase (NAG) and $\beta_{2}$-microglobulin, calcium, phosphate, magnesium, uric acid, amino acids, and malondialdehyde derived from the destruction of membrane lipids by peroxidation (Table 1).,4,6,31-33 These markers, when compared to age-

Table 1. Frequency of positive renal biomarkers throughout studies in TDT, organized by renal injury types.

\begin{tabular}{|c|c|}
\hline Renal Biomarkers & Range of frequency (\%) \\
\hline \multicolumn{2}{|c|}{ Markers of renal tubular injury } \\
\hline Hyperphosphaturia & $0-9.2$ \\
\hline Hypercalciuria & $0-79.2$ \\
\hline Aminoaciduria & $5.9-31.4$ \\
\hline Magnesiumuria & 8.6 \\
\hline Hyperaciduricuria & $9.9-82.4$ \\
\hline Urinary 2-Microglobulin & $0-64.6$ \\
\hline Elevated FENa & $0-29.1$ \\
\hline Urinary N-acetyl- -D-glucosaminidase (NAG) & $35.9-100$ \\
\hline Elevated Serum 2-Microglobulin & 5 \\
\hline Elevated urinary retinol binding protein (RBP) & 69.4 \\
\hline Elevated urinary alpha-1 Macroglobulin & 54.8 \\
\hline Decreased urinary osmolarity & $58.1-100$ \\
\hline \multicolumn{2}{|c|}{ Markers of glomerular injury and GFR } \\
\hline Elevated serum Cystatin-C & $33.2-50$ \\
\hline Glomerular hyperfiltration by eGFR & 17.8-39.6 \\
\hline Decreased eGFR & $0-58.82$ \\
\hline \multicolumn{2}{|c|}{ Mixed glomerular/Tubular injury } \\
\hline Proteinuria & $0-90$ \\
\hline Microalbumiruia & $0-100$ \\
\hline \multicolumn{2}{|c|}{ Others } \\
\hline Hematuria & $3.4-10.6$ \\
\hline
\end{tabular}


matched patients, are reduced in TDT patients who are cured by hematopoietic stem-cell transplantation. ${ }^{34}$

When urinary beta- 2 microglobulin to creatinine ratio (BCR) was used to predict renal tubular damage, the use of alendronate was linked to less damage with TDT and NTDT patients. This however was in patients requiring alendronate as indicated for osteoporosis, and should be interpreted carefully, as cases of high dose bisphosphonates are known to cause proximal tubular nephrotoxicity. ${ }^{28}$ When TDT and NTDT patients were compared, abnormal tubular function tests, including elevations in fractional excretion of sodium (FENa), urinary excretion of uric acid and alpha-1 macroglobulin (alpha-1M), were similar. However, retinol-binding protein (RBP), urine calcium/creatinine ratio $\left(\mathrm{U}_{\mathrm{Ca} / \mathrm{Cr}}\right)$ and $\mathrm{BCR}$ were higher in TDT while being respectively normal, normal and mildly elevated in NTDT. This might indicate that these parameters correlate better with blood transfusion, and perhaps iron overload mediated kidney damage. ${ }^{30}$ Hypercalciuria (elevated $\mathrm{U}_{\mathrm{Ca} / \mathrm{Cr}}$ ) evidently correlated with blood transfusions in a previous study in NTDT. ${ }^{35}$

\section{Glomerular injury}

Glomerular disease in thalassemia is related either to GFR or albuminuria.

\section{Changes in GFR}

Glomerular filtration rate is reportedly elevated in TDT patients as compared to age-matched healthy individuals. ${ }^{35,36}$ Glomerular hyperfiltration can range from 20 to $40 \%$ of thalassemia cases. ${ }^{28,35}$ Male gender and a previous history of splenectomy were found to be independent predictors in multivariate analyses. ${ }^{28}$ In NTDT patients, anemia may prove pivotal in the observed renal hyperfiltration. Regular blood transfusions decreased this phenomenon, but were also linked to increased hypercalciuria. This strengthens both the role of chronic anemia/hypoxia in the pathophysiology of glomerular hyperfiltraion, as well as the role of blood transfusion in some aspects of tubular dysfunction. ${ }^{35}$ Glomerular hyperfiltration might be an early hallmark of NTDT renal manifestations. A retrospective study of 50 NTDT patients revealed glomerular hyperfiltration to be present early in the course of the disease with nearly half of the patients exhibiting this finding. Importantly, age negatively correlated with GFR. Within the upcoming years, around $60 \%$ of these patients had abnormal urine protein to creatinine ratio (UPCR) of $\geq 200 \mathrm{mg} / \mathrm{g}$, and $14 \%$ developed proteinuria with UPCR $>500 \mathrm{mg} / \mathrm{g}$. Proteinuria positively correlated with higher levels of LIC and NTBI. ${ }^{29}$ However, contrary to diabetic and hypertensive glomerular diseases, little evidence exists so far to claim that hyperfiltration in thalassemia is indeed a harbinger for future renal damage, as it is merely explained by the physiological hyperdynamic compensation in anemic patients, which is understandably larger in a male habitus or in splenectomized patients. ${ }^{28}$ Long term follow-up studies may validate this assumption.

Although decreased GFR is rarely documented at pediatric age, gradual decrease can creep up with progressive renal damage, and it can start appearing in adult patients. ${ }^{37} 18.5 \%$ of adult thalassemic patients had eGFR $<90 \mathrm{~mL} / \mathrm{min}$ by the end of a 10 year follow up study. This decline in eGFR was more significant in patients with tubular damage. ${ }^{38}$ Decreasing GFR might reflect accumulating long-term damage to the kidneys. This follow-up however was in a period without proper blood transfusion guidelines, and when iron chelation was still widely inadequately used. More recent cohort studies are required.

\section{Albuminuria}

Different studies used different cut-off values of albumin to creatinine ratio (ACR), and even different biomarkers, to detected proteinuria as a marker for glomerular damage, making it difficult to interpret discrepancies. In pediatric TDT patients, proteinuria ranged from $24 \%$ to $47 \%$, and did not change with deferoxamine therapy. ${ }^{39,40}$ Significant glomerular proteinuria in adult patients was $20 \%$ using ACR, but was found to be $33 \%$ in another study using serum cystatin-C. ${ }^{28,31}$ Comparison of TDT vs NTDT revealed elevated serum cystatin-C and spot ACR levels in both groups compared to general population, but no clinical significant difference amongst them was found..$^{30}$ When ACR was used in TDT and NTDT patients, T2* value $\leq 20 \mathrm{msec}$ on cardiac magnetic resonance was the only independent predictor for glomerular dysfunction; it was not associated, however, with renal tubular damage or hyperfiltration. A possible link between cardiac disease, renal dysfunction and iron overload in thalassemia could be suggested, but this requires further targeted studies that include measurements such as NTBI or free oxygen radicals in the serum. ${ }^{28}$ There is still a debate over the link between ICT, mainly deferasirox, and glomerular disease. ${ }^{28,39}$

\section{ICT-mediated acute kidney injury}

Although rare, acute kidney injury (AKI) has been attributed to ICT. ${ }^{41,42}$ AKI has been reported in $40 \%$ of patients on DFO. This mostly occurred with the intravenous method of administration following overdose of the drug due to malfunction of the pump and/or inadequate monitoring during treatment. AKI, should it occur under such cases, appears to respond well to high-efficiency hemodialysis. ${ }^{43,44}$ Several cases of AKI have also been reported in the post marketing surveillance of DFX. ${ }^{4}$

In a multicenter randomized phase 3 trial comparing deferasirox and deferoxamine in TDT patients, a dose-dependent increase in serum creatinine was observed in $38 \%$ of patients receiving DFX, and a similar increase was observed in $14 \%$ of patients on DFO. The increases were mostly within normal range and never exceeded twice the upper limit normal (ULN). In most patients receiving DFX who experienced elevated serum creatinine, levels spontaneously normalized and thus dose reduction was only required in $13 \%$ of patients. ${ }^{25}$

To determine if the elevated creatinine level was progressive, the outcome of 1074 iron overloaded thalassemia patients treated with DFX for one year was reviewed. $10 \%$ of the patients experienced a rise in serum creatinine ( $>33 \%$ baseline or $>$ ULN) on two consecutive measurements. Most of these patients had high baseline serum creatinine levels. One case of AKI was reported. Overall, there was no progressive increase in mean serum creatinine. ${ }^{45}$ In parallel, another study that investigated cystatin C levels in TDT patients receiving DFX demonstrated stable levels over the 9 months period of the treatment. ${ }^{46}$

\section{Renal hemosiderosis}

Although iron deposition is documented in biopsied tubules of TDT patients, iron detection on magnetic resonance imaging (MRI) R2* for adult patients was closer to the healthy population group as compared to sickle cell disease (SCD) patients. The mechanism for anemia in SCD is intravascular hemolysis as compared to ineffective erythropoiesis in thalassemia; this may play a role in the difference of outcomes. ${ }^{47}$ Renal parameters, mainly markers of tubular dysfunction, have correlated, albeit conflictingly, with serum ferritin and LIC, as well as with iron chelation therapy use, suggesting a possible impact of iron kidney accumulation on the renal function. ${ }^{16,48,49}$ For example, Increases in NAG and $\beta 2$ $\mathrm{M}$ are higher in patients with high serum ferritin levels, and chelation therapy showed reversal of the findings..$^{33,49}$ More recently, $77 \%$ of a group of adult TDT patients showed evidence of hemosiderosis on kidney MRI T2*. $51.5 \%$ of the same group showed 
decreased eGFR. ${ }^{31}$ This could reflect that minimal iron accumulation can have an impact on renal function, but as more accumulation becomes detectable on MRI, kidney function can worsen. Further studies are needed to prove such correlations.

\section{End stage renal disease}

In a cohort of 127 NTDT patients, 4\% developed ESRD that required regular hemodialysis. A review of their medical charts over a 10 years observation period revealed that elevations in serum creatinine and dipstick-positive albuminuria were the first manifestations of renal disease. ${ }^{22}$ One patient with nephrotic range proteinuria was found to have focal segmental glomerulosclerosis (FSGS) on renal biopsy. Iron overload and paradoxically excessive iron removal, as well as the hemodynamic maladaptation related to anemia with resultant hyperfiltration, have all been suggested to gradually result in tubular atrophy and eventual glomerulo-interstitial fibrosis. $., 6,38,50$

\section{Fanconi sydrome}

Renal wasting of electolytes, amino acids and glucose occurs in thalassemia, and it is most likely a tubular dysfunction. ${ }^{33,51}$ Deferasirox, which is minimally excreted in the kidneys, is linked to fanconi syndrome (FS), a heightened form of proximal tubular wasting manifested by hypophosphatemia, normal anion gap metabolic acidosis, glucosuria, and proteinuria. During clinical trials with DFX therapy, FS was a rare complication, occurring in 0.1 to $1 \%$ of patients. ${ }^{25,52}$ It is postulated that deferasirox's lipophilic properties allow it to penetrate cell membranes and to accumulate in the proximal tubular cells. This can either cause direct nephrotoxic effects or deplete mitochondrial iron, with resulting proximal tubular dysfunction. To date, more than 14 cases of FS (mostly $\beta$ thalassemia patients) have been reported secondary to DFX therapy. ${ }^{53-55}$ Management includes discontinuation of DFX and hospitalization for fluids and electrolytes repletion. Among three re-challenge attempts, one patient had recurrent tubulopathy leading to permanent discontinuation, while the other two required long-term electrolyte supplementation when restarted on a lower dose. ${ }^{56,57}$ Plasmapheresis did not appear to be helpful in a case of acute deferasirox toxicity. ${ }^{55}$ Monitoring guidelines for DFX therapy do not suggest monthly testing for serum electrolytes and urine analysis, and therefore detection is delayed until patients are symptomatic and need hospitalization for management. Given that most patients affected with DFX tubulopathy are at the higher end of the dosing range, it is advised to check serum electrolytes at baseline and every 3 months following initiation of therapy. Prompt withdrawal of DFX and referral to a nephrologist is advised with laboratory evidence of electrolyte abnormalities. ${ }^{58}$

\section{Urolithiasis}

Transfusion-dependent thalassemia patients have a higher occurrence of both asymptomatic (59\%) and symptomatic $(18 \%)$ kidney stones compared to the general population. Stones have a mixed composition but they are most commonly calcium based. The latter are associated with lower femoral z-scores on bone marrow densitometry; calcium stone formers were also found to have lower ferritin levels and higher serum creatinine levels. ${ }^{59}$ Hypercalciuria and proteinuria also commonly co-exist with urolithiasis, and hypercalciuria strongly correlated with deferasirox body-adjusted dose, paralleling previous studies. ${ }^{60}$ Data from a 19-year longitudinal study shows that a switch from deferoxamine to deferasirox is associated with faster bone resorption. ${ }^{61}$ All these notions hint at deferasirox use as a link between bone and kidney manifestations in thalassemia. Among other stone types, thalassemic patients frequently form cysteine stones, and the reason could lie in an underlying tubulopathy. Moreover, the occurrence of struvite stones may warrant searching for undetected chronic kidney infections, especially in the splenectomized patients. ${ }^{59}$ Hematuria has ranged from 3.4 to $10.6 \%$ in observational studies on TDT and NTDT patients, and it is mostly attributable to asymptomatic kidney stones. ${ }^{32,62}$ Splenectomy, with the subsequent high erythrocyte turnover, is associated with higher rates of nephrolithiasis in NTDT. ${ }^{63}$

\section{Detection, prevention and management}

There is a trend towards earlier detection of glomerular and tubular abnormalities, especially in pediatric thalassemic populations, using early biomarkers of renal dysfunction. Table 1 and supplement 1 report the frequency and range of abnormal renal biomarkers from studies on thalassemia variants. ${ }^{15,16,27-33,35,36,39,40,51,59,64-72}$ ACR and BCR can predict initial signs of glomerular and tubular impairment, respectively, in the general population; their use in many aforementioned studies reflects their validity in the thalassemic population. ${ }^{28,73}$ Serum cystatin-C and serum or urinary beta- 2 microglobulin have received attention as better tools to assess sensitive changes GFR and creatinine clearance in thalassemia as compared to serum creatinine and eGFR. ${ }^{16,39,46,72,74-76}$ Serum cystatin-C is filtered by the glomerulus, but is neither secreted nor reabsorbed by tubular cells, so it is also a reliable marker for glomerular dysfunction, while beta- 2 microglobulin is filtered but almost completely reabsorbed by the tubules, giving its importance in screening for tubular abnormalities. ${ }^{39}$ It seems that these markers may independently correlate with serum ferritin, transfusion rate, duration of chelation therapy, albumin/creatinine ratio, serum creatinine, LIC and age, while also negatively correlating with eGFR, creatinine clearance, and hemoglobin. ${ }^{21,22,35}$ Serum cystatin-C is high in thalassemia minor but higher in NTDT and TDT groups as compared to healthy patients; this suggests its possible value as an early precursor of glomerular dysfunction in patients with thalassemia. ${ }^{30} \mathrm{NAG}$ is another marker worth use as it correlates with proteinuria..$^{27,72,77}$ Hypercalciuria, tested by urine calcium to creatinine ratio, is consistent within many studies with proximal tubulopathy. It correlates best with blood transfusion burden and deferasirox body-adjusted dose. ${ }^{31,35,40,59,61,68}$ But it could also be found in patients who are not blood transfused and should be carefully assessed with concomitant mineral bone diseases. ${ }^{30,33,68,78,79}$ Conflicting data prompt creating meta-analyses which can provide conclusive correlations and strong diagnostic statistical parameters for these biomarkers; ${ }^{28,36,46,76,80}$ However, these are difficult to achieve due to many reasons (Table 2). UPCR remains an important and frequently used marker for glomerular dysfunction. ${ }^{30,68,78}$

eGFR (using the Schwartz Formula) might not be an accurate assessment in thalassemia, as some studies suggest it overestimates the actual filtration rates when compared to 24-hour urine creatinine collections, especially in states of hyperfiltration. ${ }^{27,35,67}$ eGFR and serum ferritin have been correlated with tests like FENa, beta2 microglobulin, UPCR and cystatin-c, but alone they are not good enough predictors of renal outcomes..$^{28,30}$

Studies show opposing relationships between FENa levels and renal disease severity in beta thalassemia patients. ${ }^{27,30,33,68,76,81}$ Age difference of study groups and its implication on renal dysfunction might play a role in the different results, but systemic analysis is needed to prove this.

Urinary RBP is a specific marker for tubular dysfunction, even in the presence of massive proteinuria. Alpha-1M and RBP still have limited data in thalassemia screening for renal function, but they do show reliable results. RBP stands out in being elevated only in TDT as compared to other factors that are equally elevated in NTDT as well. ${ }^{30,82}$ 
Table 2. Factors hindering proper meta-analyses for renal biomarkers in thalassemia studies. Factors affecting proper meta-analysis construction for renal biomarkers in thalassemia

\begin{tabular}{l} 
Majority of studies have small sample size $(<10$ to 500$)$ \\
Different parameters and definitions are used for different renal injury markers \\
Different techniques are used to detect same biomarkers across studies \\
\hline Populations studied are not homogeneous (age, ICT use, transfusion burden, splenectomy, hemoglobin levels, iron overload status) \\
Studies do not account for concomitant medication (calcium, vitamin D, ICT, etc.) \\
Observational studies present data in different methods (incidence of abnormalities $v$ s average value of tests), affecting uniform information collection \\
Other kidney diseases can affect results and not always accounted for (hep C, diabetes, etc.)
\end{tabular}

Table 3. Guidance for management of renal issues with iron chelation therapy.

\begin{tabular}{|c|c|c|c|}
\hline Issue & DFO & DFP & DFX \\
\hline Pre-cautionary & $\begin{array}{l}\text { Monitor patients for changes } \\
\text { in renal function }\end{array}$ & $\begin{array}{l}\text { Special care must be taken in } \\
\text { patients with renal impairment } \\
\text { since studies have not been } \\
\text { conducted to evaluate its safety } \\
\text { and efficacy in these patients }\end{array}$ & $\begin{array}{l}\text { Measure serum creatinine and } \mathrm{CrCl} \text { before } \\
\text { starting therapy; Monitor serum creatinine } \\
\text { regularly }\end{array}$ \\
\hline Baseline renal impairment & $\begin{array}{l}\text { Contraindicated in patients with } \\
\text { severe renal disease or anuria }\end{array}$ & $\begin{array}{l}\text { DFP has not been evaluated in } \\
\text { patients with renal impairment }\end{array}$ & $\begin{array}{l}\text { Impairment of } \mathrm{CrCl} 40-60 \mathrm{~mL} / \mathrm{min} \text { : reduce } \\
\text { starting dose by } 50 \% \text {; do not use if serum } \\
\text { creatinine }>2 \times \mathrm{ULN} \text { or } \mathrm{CrCl}<40 \mathrm{~mL} / \mathrm{min}\end{array}$ \\
\hline Increased serum creatinine & $\begin{array}{l}\text { Monitor patients for changes in } \\
\text { renal function; increased serum } \\
\text { creatinine, acute renal failure, and } \\
\text { renal tubular disorders have been } \\
\text { reported in postmarketing studies }\end{array}$ & (No guidance provided) & $\begin{array}{l}\text { Treatment should be interrupted or reduced } \\
\text { if increase is }>33 \% \text { above baseline at } 2 \\
\text { consecutive visits (and above the age-appropriate } \\
\text { ULN in pediatric patients) }\end{array}$ \\
\hline Continued renal impairment & (No guidance provided) & (No guidance provided) & $\begin{array}{l}\text { Discontinue therapy if } \mathrm{CrCl}<40 \mathrm{~mL} / \mathrm{min} \text {, or serum } \\
\text { creatinine } \\
>2 \times \text { age-appropriate ULN }\end{array}$ \\
\hline
\end{tabular}

So far, according to the thalassemia international federation (TIF) guidelines of 2014 for TDT management, periodic chemistry panel, urea and creatinine should be done every 3 months (monthly if on DFX). Urinalysis is to be done biannually. ${ }^{3}$ Since iron overload, chronic anemia and improper ICT use are all linked to renal complications, the judicial use of blood transfusion and iron chelators, along with proper follow-up testing, is recommended. It is always important to rule out and manage other diseases that can affect the kidneys, such as diabetes, hypertension, etc. ${ }^{4}$ Table 3 aids in the choice and monitoring recommendations when using iron chelation therapy. ${ }^{83-85}$

\section{Conclusions}

Renal disease is a long-term complication that should be recognized in thalassemia, especially with the rise in the average age of this population. Proper assessment of renal function abnormalities in thalassemia can be challenging because of the increased use of iron chelators, which themselves can affect renal function. With increasing information concerning the renal abnormalities and convenient renal biomarkers, good meta-analyses can shed light on the best tools to use for assessment and prevention of renal disease in thalassemic patients. However, definitions and markers of kidney disease across the studies are highly heterogeneous, and most studies available are cross-sectional, involving a small number of patients. More longitudinal data is required to fully portray any possible differences between TDT and NTDT abnormalities, as well the current prevalence of these diseases in the era of new iron chelation and blood transfusion guidelines. With enough data still lacking, there is a need for close monitoring and follow-up of renal function in NTDT patients as they live longer, and this puts them at increased risk of severe renal disease. Progress of research in this topic will allow the detection of renal dysfunction harbingers in hope to arrest the progress of renal injury, if not to reverse it.

\section{References}

1. Taher AT, Weatherall DJ and Cappellini MD. Thalassaemia. Lancet 2017. DOI: 10.1016/S0140-6736(17)31822-6.

2. Sleiman J, Tarhini A, Bou-Fakhredin R, et al. Non-transfusiondependent thalassemia: an update on complications and management. INT J MOL SCI 2018; 19: 182.

3. Cappellini MD, Cohen A, Porter J, et al. Guidelines for the management of transfusion dependent thalassaemia (TDT). Nicosia (CY): Thalassaemia International Federation, 2014.

4. Ponticelli C, Musallam KM, Cianciulli P, et al. Renal complications in transfusion-dependent beta thalassaemia. Blood reviews 2010; 24: 239-244. DOI: 10.1016/j.blre.2010.08.004.

5. Mallat NS, Mallat SG, Musallam KM, et al. Potential mechanisms for renal damage in beta-thalassemia. J Nephrol 2013; 26: 821-828. DOI: 10.5301/jn.5000253.

6. Musallam KM and Taher AT. Mechanisms of renal disease in beta-thalassemia. Journal of the American Society of 
Nephrology: JASN 2012; 23: 1299-1302. DOI: 10.1681/ASN. 2011111070.

7. Landing BH, Gonick HC, Nadorra RL, et al. Renal lesions and clinical findings in thalassemia major and other chronic anemias with hemosiderosis. Pediatric pathology 1989; 9: 479-500.

8. Martines AM, Masereeuw R, Tjalsma H, et al. Iron metabolism in the pathogenesis of iron-induced kidney injury. Nature reviews Nephrology 2013; 9: 385-398. DOI: 10.1038/nrneph. 2013.98.

9. Kokoszko A, Dabrowski J, Lewinski A, et al. Protective effects of GH and IGF-I against iron-induced lipid peroxidation in vivo. Experimental and toxicologic pathology : official journal of the Gesellschaft fur Toxikologische Pathologie 2008; 60: 453-458. DOI: 10.1016/j.etp.2008.04.012.

10. Kassab-Chekir A, Laradi S, Ferchichi S, et al. Oxidant, antioxidant status and metabolic data in patients with beta-thalassemia. Clinica chimica acta; international journal of clinical chemistry 2003; 338: 79-86.

11. Zager RA, Johnson AC and Hanson SY. Parenteral iron nephrotoxicity: potential mechanisms and consequences. Kidney international 2004; 66: 144-156. DOI: 10.1111/j.15231755.2004.00716.x.

12. Shah SV. Oxidants and iron in chronic kidney disease. Kidney international Supplement 2004: S50.

13. Alfrey AC. Role of iron and oxygen radicals in the progression of chronic renal failure. American journal of kidney diseases : the official journal of the National Kidney Foundation 1994; 23: 183-187.

14. Fibach $\mathrm{E}$ and Rachmilewitz E. The role of oxidative stress in hemolytic anemia. Current molecular medicine 2008; 8: 609619.

15. Sumboonnanonda A, Malasit P, Tanphaichitr VS, et al. Renal tubular function in beta-thalassemia. Pediatric nephrology 1998; 12: 280-283.

16. Koliakos G, Papachristou F, Koussi A, et al. Urine biochemical markers of early renal dysfunction are associated with iron overload in beta-thalassaemia. Clinical and laboratory haematology 2003; 25: 105-109.

17. Davis LE and Hohimer AR. Hemodynamics and organ blood flow in fetal sheep subjected to chronic anemia. The American journal of physiology 1991; 261: R1542-1548. DOI: 10.1152/ajpregu.1991.261.6.R1542.

18. Palatini P. Glomerular hyperfiltration: a marker of early renal damage in pre-diabetes and pre-hypertension. Nephrology, dialysis, transplantation : official publication of the European Dialysis and Transplant Association - European Renal Association 2012; 27: 1708-1714. DOI: 10.1093/ndt/gfs037.

19. Lafferty HM, Anderson S and Brenner BM. Anemia: a potent modulator of renal hemodynamics in models of progressive renal disease. American journal of kidney diseases : the official journal of the National Kidney Foundation 1991; 17: 2-7.

20. Nangaku M. Chronic hypoxia and tubulointerstitial injury: a final common pathway to end-stage renal failure. Journal of the American Society of Nephrology : JASN 2006; 17: 17-25. DOI: 10.1681/ASN.2005070757.

21. Brenner BM, Lawler EV and Mackenzie HS. The hyperfiltration theory: a paradigm shift in nephrology. Kidney international 1996; 49: 1774-1777.

22. Mallat NS, Musallam KM, Mallat SG, et al. End stage renal disease in six patients with beta-thalassemia intermedia. Blood cells, molecules \& diseases 2013; 51: 146-148. DOI: 10.1016/ j.bcmd.2013.05.001.

23. Cianciulli P, Sollecito D, Sorrentino F, et al. Early detection of nephrotoxic effects in thalassemic patients receiving desferrioxamine therapy. Kidney international 1994; 46: 467-470.

24. Clajus C, Becker JU, Stichtenoth DO, et al. Acute kidney injury due to deferoxamine in a renal transplant patient. Nephrology, dialysis, transplantation : official publication of the European Dialysis and Transplant Association - European Renal Association 2008; 23: 1061-1064. DOI: 10.1093/ndt/ gfm824.

25. Cappellini MD, Cohen A, Piga A, et al. A phase 3 study of deferasirox (ICL670), a once-daily oral iron chelator, in patients with beta-thalassemia. Blood 2006; 107: 3455-3462. DOI: 10.1182 /blood-2005-08-3430.

26. Vichinsky E, Onyekwere O, Porter J, et al. A randomised comparison of deferasirox versus deferoxamine for the treatment of transfusional iron overload in sickle cell disease. British journal of haematology 2007; 136: 501-508. DOI: 10.1111/j.13652141.2006.06455.x.

27. Jalali A, Khalilian H, Ahmadzadeh A, et al. Renal function in transfusion-dependent pediatric beta-thalassemia major patients. Hematology 2011; 16: 249-254. DOI: 10.1179/ 102453311 X12953015767662.

28. Deveci B, Kurtoglu A, Kurtoglu E, et al. Documentation of renal glomerular and tubular impairment and glomerular hyperfiltration in multitransfused patients with beta thalassemia. Annals of hematology 2016; 95: 375-381. DOI: 10.1007/s00277-015-2561-2.

29. Ziyadeh FN, Musallam KM, Mallat NS, et al. Glomerular hyperfiltration and proteinuria in transfusion-independent patients with beta-thalassemia intermedia. Nephron Clin Pract 2012; 121: c136-143. DOI: 10.1159/000339787.

30. Uzun E, Balci YI, Yuksel S, et al. Glomerular and tubular functions in children with different forms of beta thalassemia. Renal failure 2015; 37: 1414-1418. DOI: 10.3109/0886022X. 2015.1077314.

31. Hashemieh M, Radfar M, Azarkeivan A, et al. Renal hemosiderosis among iranian transfusion dependent $\beta$-thalassemia major patients. International Journal of Hematology-Oncology and Stem Cell Research 2017; 11: 133-138.

32. Ahmadzadeh A, Jalali A, Assar S, et al. Renal tubular dysfunction in pediatric patients with beta-thalassemia major. Saudi J Kidney Dis Transpl 2011; 22: 497-500.

33. Smolkin V, Halevy R, Levin C, et al. Renal function in children with beta-thalassemia major and thalassemia intermedia. Pediatric nephrology 2008; 23: 1847-1851. DOI: 10.1007/ s00467-008-0897-8.

34. Sumboonnanonda A, Sanpakit K and Piyaphanee N. Renal tubule function in beta-thalassemia after hematopoietic stem cell transplantation. Pediatric nephrology 2009; 24: 183-187. DOI: 10.1007/s00467-008-0949-0.

35. Quinn CT, Johnson VL, Kim HY, et al. Renal dysfunction in patients with thalassaemia. British journal of haematology 2011; 153: 111-117. DOI: 10.1111/j.1365-2141.2010.08477.x.

36. Annayev A, Karakas Z, Karaman S, et al. Glomerular and tubular functions in children and adults with transfusion dependent thalassemia. Turkish journal of haematology : official journal of Turkish Society of Haematology 2017. DOI: 10.4274/tjh.2017.0266.

37. Behairy OG, Abd Almonaem ER, Abed NT, et al. Role of serum cystatin- $\mathrm{C}$ and beta-2 microglobulin as early markers of renal dysfunction in children with beta thalassemia major. International journal of nephrology and renovascular disease 2017; 10: 261-268. DOI: 10.2147/IJNRD.S142824.

38. Lai ME, Spiga A, Vacquer S, et al. Renal function in patients with beta-thalassaemia major: a long-term follow-up study. Nephrology, dialysis, transplantation : official publication of the 
European Dialysis and Transplant Association - European Renal Association 2012; 27: 3547-3551. DOI: 10.1093/ndt/gfs169.

39. Hamed EA and ElMelegy NT. Renal functions in pediatric patients with beta-thalassemia major: relation to chelation therapy: original prospective study. Italian journal of pediatrics 2010; 36: 39. DOI: 10.1186/1824-7288-36-39.

40. Economou M, Printza N, Teli A, et al. Renal dysfunction in patients with beta-thalassemia major receiving iron chelation therapy either with deferoxamine and deferiprone or with deferasirox. Acta haematologica 2010; 123: 148-152. DOI: $10.1159 / 000287238$.

41. Koren G, Bentur Y, Strong D, et al. Acute changes in renal function associated with deferoxamine therapy. Am J Dis Child 1989; 143: 1077-1080.

42. Cianciulli P, Sorrentino F, Forte L, et al. Acute renal failure occurring during intravenous desferrioxamine therapy: recovery after haemodialysis. Haematologica 1992; 77: 514-515.

43. Li Volti S, Maccarone C, Li Volti G, et al. Acute renal failure following deferoxamine overdose. Pediatric nephrology 2003; 18: 1078-1079. DOI: 10.1007/s00467-003-1249-3.

44. Prasannan L, Flynn JT and Levine JE. Acute renal failure following deferoxamine overdose. Pediatric nephrology 2003; 18: 283-285. DOI: $10.1007 / \mathrm{s} 00467-002-1051-7$.

45. Cappellini MD and Taher A. Deferasirox (Exjade) for the treatment of iron overload. Acta haematologica 2009; 122: 165173. DOI: $10.1159 / 000243801$

46. Papassotiriou I, Margeli A, Hantzi E, et al. Cystatin C levels in patients with beta-thalassemia during deferasirox treatment. Blood cells, molecules \& diseases 2010; 44: 152-155. DOI: 10.1016/j.bcmd.2010.01.001.

47. Schein A, Enriquez C, Coates TD, et al. Magnetic resonance detection of kidney iron deposition in sickle cell disease: a marker of chronic hemolysis. Journal of magnetic resonance imaging: JMRI 2008; 28: 698-704. DOI: 10.1002/ jmri.21490.

48. Hashemieh M, Azarkeivan A, Akhlaghpoor S, et al. T2-star $(\mathrm{T} 2 *)$ magnetic resonance imaging for assessment of kidney iron overload in thalassemic patients. Archives of Iranian medicine 2012; 15: 91-94. DOI: 012152/AIM.009.

49. Michelakakis H, Dimitriou E, Georgakis $\mathrm{H}$, et al. Iron overload and urinary lysosomal enzyme levels in beta-thalassaemia major. European journal of pediatrics 1997; 156: 602-604.

50. D'Agati VD, Kaskel FJ and Falk RJ. Focal segmental glomerulosclerosis. The New England journal of medicine 2011; 365: 2398-2411. DOI: 10.1056/NEJMra1106556.

51. Sadeghi-Bojd S, Hashemi M and Karimi M. Renal tubular function in patients with beta-thalassaemia major in zahedan, southeast iran. Singapore Med J 2008; 49: 410-412.

52. Cappellini MD, Bejaoui M, Agaoglu L, et al. Iron chelation with deferasirox in adult and pediatric patients with thalassemia major: efficacy and safety during 5 years' follow-up. Blood 2011;118: 884-893. DOI: 10.1182/blood-2010-11-316646.

53. Rheault MN, Bechtel H, Neglia JP, et al. Reversible fanconi syndrome in a pediatric patient on deferasirox. Pediatric blood \& cancer 2011; 56: 674-676. DOI: 10.1002/pbc.22711.

54. Dee CM, Cheuk DK, Ha SY, et al. Incidence of deferasiroxassociated renal tubular dysfunction in children and young adults with beta-thalassaemia. Br J Haematol 2014; 167: 434436. DOI: $10.1111 /$ bjh. 13002 .

55. Shah L, Powell JL and Zaritsky JJ. A case of Fanconi syndrome due to a deferasirox overdose and a trial of plasmapheresis. Journal of clinical pharmacy and therapeutics 2017; 42: 634-637. DOI: $10.1111 /$ jept.12553.

56. Even-Or E, Becker-Cohen R and Miskin H. Deferasirox treat- ment may be associated with reversible renal Fanconi syndrome. American journal of hematology 2010; 85: 132-134. DOI: $10.1002 /$ ajh.21588.

57. Wei HY, Yang CP, Cheng $\mathrm{CH}$, et al. Fanconi syndrome in a patient with beta-thalassemia major after using deferasirox for 27 months. Transfusion 2011; 51: 949-954. DOI: 10.1111/j. 1537-2995.2010.02939.x.

58. Papneja K, Bhatt MD, Kirby-Allen M, et al. Fanconi syndrome secondary to deferasirox in diamond-blackfan anemia: case series and recommendations for early diagnosis. Pediatric blood \& cancer 2016; 63: 1480-1483. DOI: 10.1002/pbc.25995.

59. Wong P, Milat F, Fuller PJ, et al. Urolithiasis is prevalent and associated with reduced bone mineral density in beta-thalassaemia major. Internal medicine journal 2017; 47: 1064-1067. 2017/09/12. DOI: 10.1111/imj.13533.

60. Wong P, Polkinghorne K, Kerr PG, et al. Deferasirox at therapeutic doses is associated with dose-dependent hypercalciuria. Bone 2016; 85: 55-58. DOI: 10.1016/j.bone.2016.01.011.

61. Wong P, Fuller PJ, Gillespie MT, et al. Thalassemia bone disease: a 19-year longitudinal analysis. J Bone Miner Res 2014; 29: 2468-2473. DOI: 10.1002/jbmr.2266.

62. Bakr A, Al-Tonbary Y, Osman G, et al. Renal complications of beta-thalassemia major in children. Am J Blood Res 2014; 4: 1-6.

63. Ricchi P, Ammirabile M, Costantini S, et al. Splenectomy is a risk factor for developing hyperuricemia and nephrolithiasis in patients with thalassemia intermedia: a retrospective study. Blood cells, molecules \& diseases 2012; 49: 133-135. DOI: 10.1016/j.bcmd.2012.05.012.

64. Ali D, Mehran K and Moghaddam AG. Comparative evaluation of renal findings in Beta-thalassemia major and intermedia. Saudi J Kidney Dis Transpl 2008; 19: 206-209.

65. Fallahzadeh MH, Fallahzadeh MK, Shahriari M, et al. Hematuria in patients with Beta-thalassemia major. Iranian journal of kidney diseases 2010; 4: 133-136.

66. Mastrangelo F, Lopez T, Rizzelli S, et al. Function of the kidney in adult patients with Cooley's disease. A preliminary report. Nephron 1975; 14: 229-236.

67. Milo G, Feige Gross Nevo R, Pazgal I, et al. GFR in patients with beta-thalassemia major. Clin J Am Soc Nephrol 2015; 10 : 1350-1356. DOI: $10.2215 /$ CJN.12181214.

68. Mohkam M, Shamsian BS, Gharib A, et al. Early markers of renal dysfunction in patients with beta-thalassemia major. Pediatric nephrology 2008; 23: 971-976. DOI: 10.1007/ s00467-008-0753-x.

69. Mula-Abed WA, Al-Hashmi HS and Al-Muslahi MN. Indicators of Renal Glomerular and Tubular Functions in Patients with Beta-Thalassaemia Major: A cross sectional study at the Royal Hospital, Oman. Sultan Qaboos University medical journal 2011; 11: 69-76.

70. Ong-ajyooth L, Malasit P, Ong-ajyooth S, et al. Renal function in adult beta-thalassemia/Hb E disease. Nephron 1998; 78: 156-161.

71. Sumboonnanonda A, Malasit P, Tanphaichitr VS, et al. Renal tubular dysfunction in alpha-thalassemia. Pediatric nephrology 2003; 18: 257-260. DOI: 10.1007/s00467-003-1067-7.

72. Tantawy AA, El Bablawy N, Adly AA, et al. Early predictors of renal dysfunction in egyptian patients with beta-thalassemia major and intermedia. Mediterranean journal of hematology and infectious diseases 2014; 6: e2014057. DOI: 10.4084/ MJHID.2014.057.

73. Ikeda A, Konta T, Takasaki S, et al. In a non-diabetic Japanese population, the combination of macroalbuminuria and increased urine beta 2-microglobulin predicts a decline of renal function: the Takahata study. Nephrology, dialysis, transplan- 
tation : official publication of the European Dialysis and Transplant Association - European Renal Association 2009; 24: 841-847. DOI: 10.1093/ndt/gfn591.

74. Ali BA and Mahmoud AM. Frequency of glomerular dysfunction in children with Beta thalassaemia major. Sultan Qaboos University medical journal 2014; 14: e88-94.

75. Kacar A. Levels of beta- 2 microglobulin and cystatin $\mathrm{C}$ in beta thalassemia major patients. Journal of Clinical and Analytical Medicine 2014; 6: 269-273. DOI: 10.4328/JCAM.1968.

76. Jafari HM, Vahidshahi K, Kosaryan M, et al. Major beta-thalassemia, use of desferiexamine and renal proximal tubular damage. Bratislavske lekarske listy 2011; 112: 278-281. 2011/06/21.

77. Voskaridou E, Terpos E, Michail S, et al. Early markers of renal dysfunction in patients with sickle cell/beta-thalassemia. Kidney international 2006; 69: 2037-2042. DOI: 10.1038/ sj.ki.5000248.

78. Kalman S, Atay AA, Sakallioglu O, et al. Renal tubular function in children with beta-thalassemia minor. Nephrology 2005; 10: 427-429. DOI: 10.1111/j.1440-1797.2005.00484.x.

79. Cetin T, Oktenli C, Ozgurtas T, et al. Renal tubular dysfunction in beta-thalassemia minor. American journal of kidney dis- eases : the official journal of the National Kidney Foundation 2003; 42: 1164-1168.

80. Al-Khabori M, Bhandari S, Al-Rasadi K, et al. Correlation of iron overload and glomerular filtration rate estimated by cystatin $\mathrm{C}$ in patients with beta-thalassemia major. Hemoglobin 2014; 38: 365-368. DOI: 10.3109/03630269.2014.944314.

81. Aldudak B, Karabay Bayazit A, Noyan A, et al. Renal function in pediatric patients with beta-thalassemia major. Pediatric nephrology 2000; 15: 109-112.

82. Kattamis C, Lazaropoulou C, Delaporta P, et al. Disturbances of biomarkers of iron and oxidant-antioxidant homeostasis in patients with beta-thalassemia intermedia. Pediatric endocrinology reviews : PER 2011; 8 Suppl 2: 256-262.

83. Desferal ${ }^{\circledR}$ (deferoxamine mesylate): prescribing information 2011, https://www.pharma.us.novartis.com/sites/www.pharma. us.novartis.com/files/desferal.pdf (accessed January 11 2018).

84. Ferriprox ${ }^{\circledR}$ (deferiprone) prescribing information 2012, https://hemonc.org/w/images/6/6c/Deferiprone.pdf (accessed January 11 2018).

85. EXJADE® (deferasirox): prescribing information 2013, https:/www.accessdata.fda.gov/drugsatfda_docs/label/2013/0 21882s0191bl.pdf (accessed January 11 2018). 